\title{
Intelligent agent based-Real time monitoring and Evaluation of GSM quality of service
}

\author{
${ }^{1}$ Anthony Lordson Amana, \\ Veritas University, Abuja, Nigeria \\ viclordson@gmail.com \\ ${ }^{2}$ Ukeje Emmanuel, \\ University of Port Harcourt, Rivers State, Nigeria. \\ eukeje@gmail.com \\ ${ }^{3}$ Ngang Bassey Ngang, \\ Enugu State University of Science and Technology, Enugu (ESUT), Nigeria. \\ Corresponding Author: nbngang@gmail.com.
}

\begin{abstract}
Most Telecommunication companies could not guarantee complete free and quality communication network service. In recent times there had been Undesirable service delivery, this problem is traced to not having free and quality network which had arisen as a result of not monitoring when there is high bit error rate. The problem had been addressed and a solution proffered in this paper by Real time Evaluation and Monitoring of GSM quality of service utilizing intelligent agent device. This was achieved by first determining the channel capacity for GSM quality network, obtaining the bit error rate for the determined capacity, designing an intelligent GSM rule that will monitor and minimize the high bit error rate for quality service and finally designing a Simulink model for real time monitoring and evaluation of GSM quality of service using intelligent agent. The result obtained was $10 \%$ better than using the conventional methods or techniques like Hieratical, Proportional Integral Derivative (PID). Real time monitoring and Evaluation of GSM quality of service using intelligent agent is preferred to other conventional methods as it can guarantee a perfect and free network in our communication network.
\end{abstract}

Key words: Real time monitoring and evaluation of GSM quality of service, intelligent agent

DOI: $10.7176 / \mathrm{IKM} / 11-4-05$

Publication date:June $30^{\text {th }} 2021$

\section{Introduction}

The advent of modern technologies and concepts have improved the way IT services provide, maintain and regulate their services to end users. Telecommunication equipment/systems have to function to accomplish the profound effects of this new trend. Network elements starting from low resource devices to large scale distributed applications have been affected by the evolutionary trend. The new technologies and concepts in both data and telecommunication industry are modifying the way service providers, maintain and use the various IT services. Management systems have to ensure the deliverance of good services despite the effects of this technological evolution that was introduced into the network. Large quantities of different network elements ranging from low resource devices to large scale distributed applications are now in use competitively. Network congestion in data networking and queuing concept or theory has to do with reduced quality of service that occurs when a network node or link is carrying more than it can handle. The undesirable consequence is queueing delay, packet loss or the blocking of new connections eventually.

1.2 Aim of the Study

This paper is aimed at Using Intelligent Agent to achieve Real-time monitoring and evaluation of GSM quality of service in Nigeria.

\subsection{Objectives of Study}

Demand on mobile phones and data service applications have influenced the expansion of telecommunication equipment and caused Network congestion. Therefore, the objectives of this work are to

- Determine the channel capacity for GSM Quality Network.

- Evaluate the bit error rate for the determined capacity

- Design an Intelligent GSM Rule that will monitor and minimize the high bit error rate for quality Service 
- Design a SIMULINK Model for real-time monitoring and evaluation of GSM quality of service using intelligent agent.

\section{Reviews}

\subsection{Extent of Past Work}

Several studies have been carried out on GSM monitoring for good quality service. A study Performed by Sharma and Mishra [1], concentrated on Intelligent Mobile-Agent based Scalable Network Management Architecture for Large -Scale Enterprise System. In their study, they looked at the scalability and flexibility problems of centralized (SNMP or CMIP management models) models though the use of Mobile Agents to distribute and delegate management tasks. This did not address the problems affecting GSM Quality network [2][3]. Varied technologies, such as SONET, ATM, Ethernet, DWDM to mention but a few presented at different layers of the Access, Metro and Core (long haul) sections of the network, have increased the complex nature of their own framing and protocol architecture or structures. Hence, this had caused challenges in control and management of the traffic in these networks. It has been dominated by the Internet Engineering Task Force (IETF) simple network management protocol SNMP for data networks [4]. The OSI common management information protocol (CMIP) for telecommunication network [5], are typically designed according to a centralized model and hence suffer from lack of distribution, a low degree of flexibility, low scalability and fault tolerance [6][7]. These need network operators at NMS level to enhance real-time decisions and manually determine solutions for the series of problems in the network. These network management systems handles data gathering and reporting methods, which has to do with a lot of data thereby consuming a lot of bandwidth and computational overhead. In [8], It could cause a considerable strain on the network and traffic jam at the manager host. Besides This centralized management functions could be limited in their capability as their activities could be difficult, like upfront judgment, forecasting, analyzing data and make positive efforts to maintain quality of service Network management based-on Mobile agent [9] and [10] means to equip agents with network management intelligence and allowing them to send requests to controlled devices/objects after migrating close to them. Real time monitoring and Evaluation of GSM quality of service using intelligent agent is the pivot of this paper. With this it is a well-known fact that wireless mobile communication system has grown from, the first generation $(1 \mathrm{G})$ of analogue system, through the second generation $(2 \mathrm{G})$ of digital system to the ever maturing third generation (3G) high speed The Idea of agent and multi-agent based technology started from artificial intelligence $[11,12]$. Multi-agent based system is a system that is a linked network troubleshooter or solution provider that functions together to resolve technical problems or issues that are beyond the capability of a single agent [13]. Hence the roots of the concept started seventy years ago when artificial intelligence (AI) was first implemented. Software agents were first meant to be classified technique just for security reasons and are regarded as secret software detectives that provide a general computing platform for executing task like information gathering, information filtering and searching. Online shopping, personal assistant, were utilizing this technology. Multi-agent system properties and agent properties have many advantages [14, 15], they are adaptable, autonomous, pro-active and reliable. The technology was first used by the military before the before other commercial sectors. [16] designed a multi-agent system for network security management and the main area of concern in [16] was critical security management issue that is intrusion detection. While in [17], the focus was to develop a multi-agent system implementation for network management based on SNMP Protocol. The multi-agent system is made up of several agents whose purpose was to enhance the effort of network management in accordance with the established policies.

\section{Methodology}

- The methodology to achieve the aim of this study is the sequential compliance to the the research objectives step by step. Which has to do with Determination of the channel capacity for GSM Quality Network.

- Evaluation of the bit error rate for the determined capacity

- Designing an Intelligent GSM Rule that will monitor and minimize the high bit error rate for quality Service and the design of a SIMULINK Model for real-time monitoring and evaluation of GSM quality of service using intelligent agent.

\subsection{To evaluate bit error rate in channel capacity at frequency diversity}

The behavior of the network was observed using the proposed mathematical model of equations below. This aided in analyzing the system performance.

What is the channel capacity for a teleprinter channel with $a 100 \mathrm{~Hz}, 200 \mathrm{~Hz} 300 \mathrm{~Hz}, 400 \mathrm{~Hz}, 500 \mathrm{~Hz}$ and $600 \mathrm{~Hz}$ bandwidth and a signal-to-noise ratio of $3 \mathrm{~dB}$ ? 
Solution:

Using Shannon's equation: $\mathrm{C}=\mathrm{B} \log _{2}(1+\mathrm{SNR})$ we have

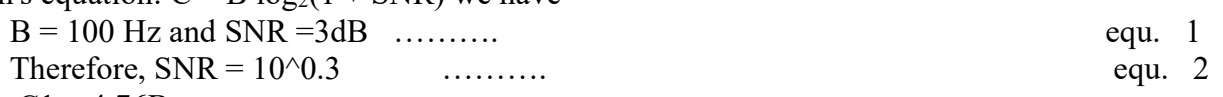

To calculate the capacity when the frequency is $200 \mathrm{~Hz}$

$$
\begin{aligned}
& \mathrm{C} 2=200 \log 2(2.995) \\
& \mathrm{C} 2=10^{\wedge} 2 * 0.476 \\
& \mathrm{C} 2=47.6 \mathrm{bps}
\end{aligned}
$$

To find the value of the capacity when the frequency is $300 \mathrm{~Hz}$

$$
\begin{aligned}
& \mathrm{B}=300 \mathrm{~Hz} \text { and } \mathrm{SNR}(\text { in } \mathrm{dB})=3, \\
& \text { Therefore, } \mathrm{SNR}=10^{0.3} \\
& \mathrm{C} 3=300 \log _{2}\left(1+10^{0.3}\right) \\
& \mathrm{C} 3=476 \mathrm{bps}
\end{aligned}
$$

To evaluate the channel capacity when the frequency is $400 \mathrm{~Hz}$

$$
\begin{aligned}
& \mathrm{C} 4=400 \log 2(2.995) \\
& \mathrm{C} 4=4760 \mathrm{bps}
\end{aligned}
$$

To solve for the channel capacity when the frequency is $500 \mathrm{~Hz}$

$$
\begin{aligned}
\mathrm{C} 5 & =500 \log 2(2.995) \\
\mathrm{C} 5 & =47600 \mathrm{bps}
\end{aligned}
$$

To calculate the channel capacity when the frequency is $600 \mathrm{~Hz}$

$$
\begin{array}{r}
\mathrm{C} 6=600 \log 2(2.995) \\
\mathrm{C} 6=476000 \mathrm{bps}
\end{array}
$$

\subsection{Evaluation of the bit error rate for the given capacity}

Let's say that 1,000 bits are transmitted, and a bit, 2 bits, 3 bits, 4 bits, 5 bits, 6 bits, 7 bits and 8 bits received are error because of some kind interference between the transmitter and receiver.

BER in this example is calculated by dividing the number of errored bits by the total number of bits transmitted

$\mathrm{BER}=\frac{1}{1000} \quad=0.001$

To find BER at 2 bit error rate

$$
\frac{\mathbf{2}}{1000000}=0.000002
$$

To find BER at 3 bit error rate

$\mathrm{BER}=\underline{\text { Error bits received }}$ 
Total number of bits

$$
\frac{3}{1000}=0.003
$$

To find BER at 4 bit error rate

$\mathrm{BER}=\underline{\text { Error bits received }}$ Total number of bits

$$
\begin{array}{ll}
\mathrm{BER}=\frac{4}{1000} & =0.004
\end{array}
$$

\subsection{Designing an intelligent GSM rule that will monitor and reduce the high bit error rate}

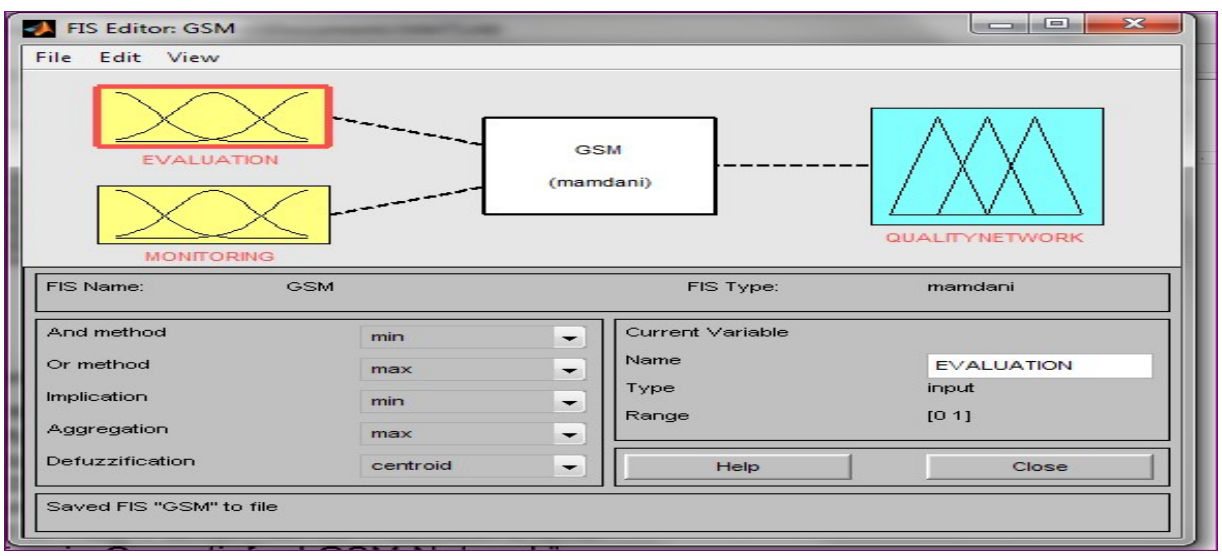

Figure 1. Fuzzy inference system editor for GSM

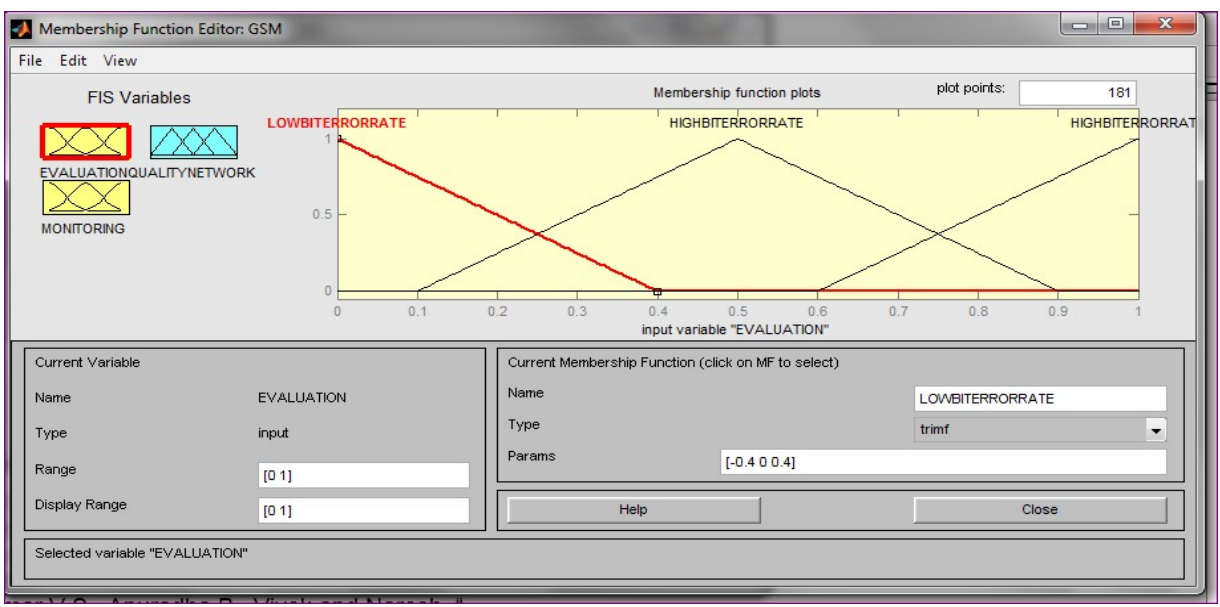

Figure 2 Membership function for GSM evaluation. 


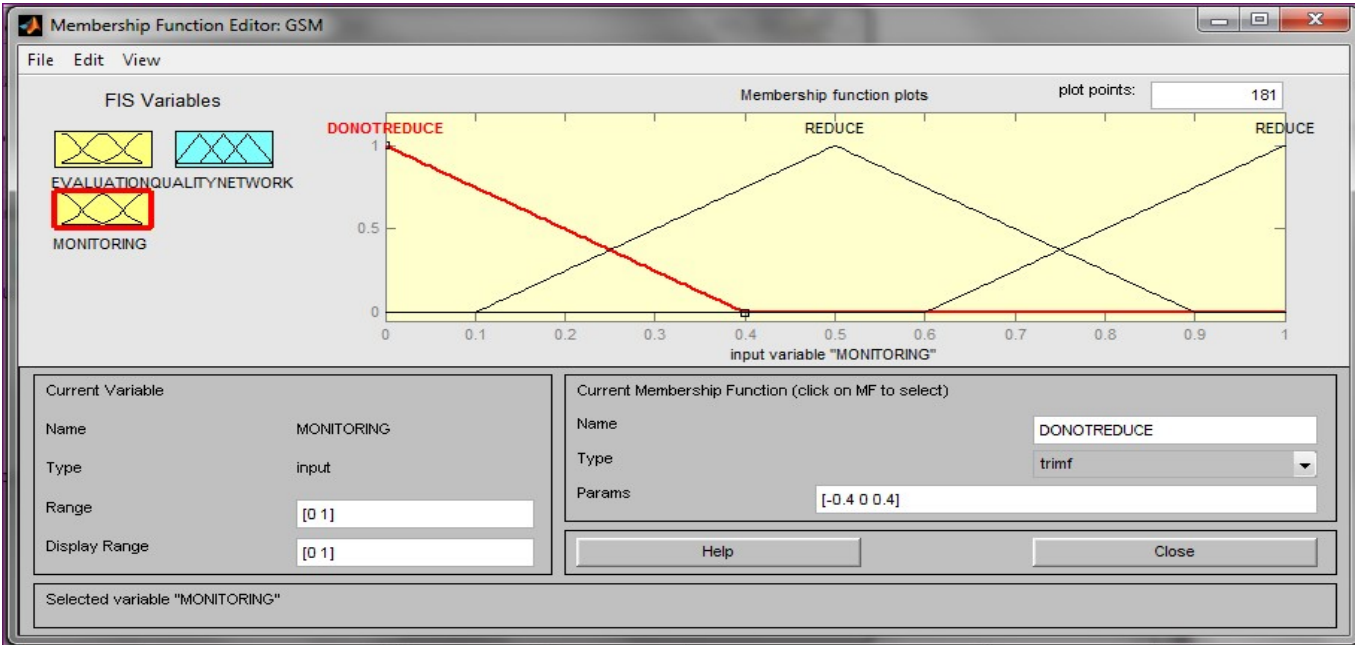

Figure 3. Membership function editor for monitoring

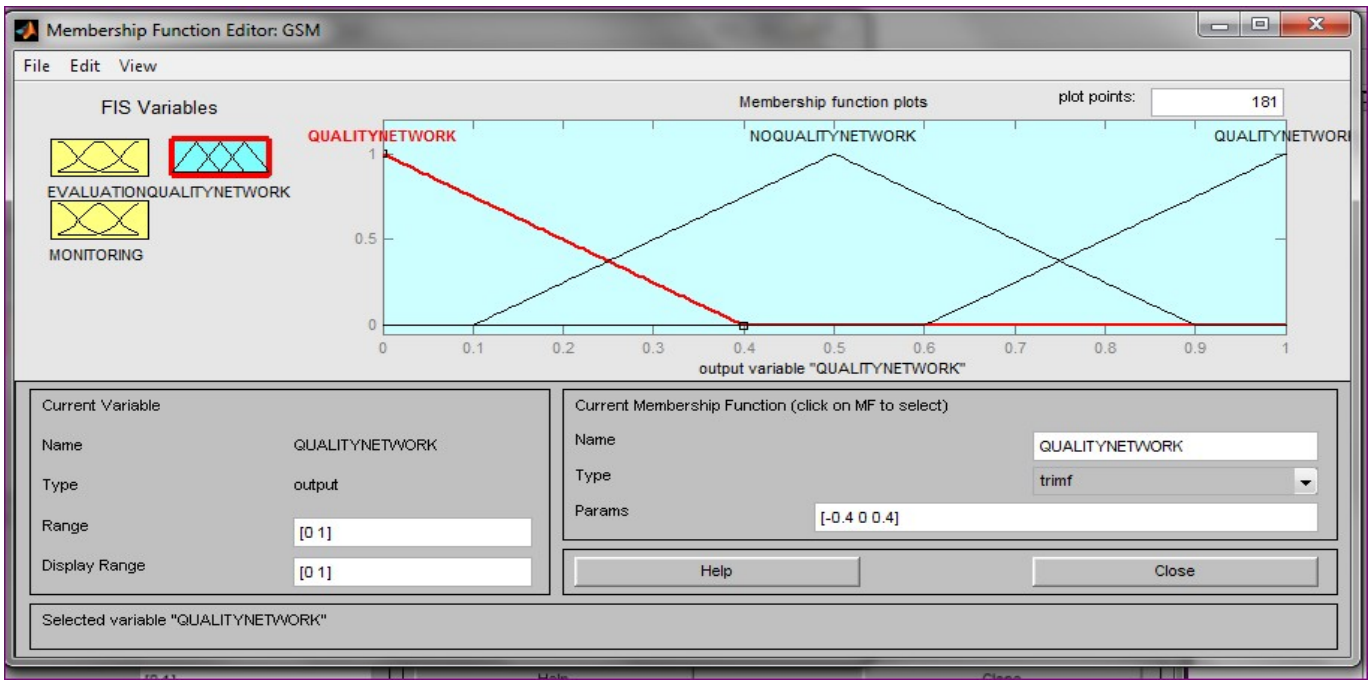

Figure 4. Membership function for quality network 
Rule Editor: GSM

File Edit View Options

1. If (EVALUATION is LOWBITERRORRATE) and (MONITORING is DONOTREDUCE) then (QUALITYNETWORF 2. If (EVALUATION is HIGHBITERRORRATE) and (MONITORING is DONOTREDUCE) then (QUALITYNETMORY 3. If (EVALUATION is HIGHBITERRORRATE) and (MONITORING is REDUCE) then (QUALITYNETMORK is QUA 4. If (EVALUATION is HIGHBITERRORRATE) and (MONITORING is DONOTREDUCE) then (QUALITYNETMORP 5. If (EVALUATION is HIGHBITERRORRATE) and (MONITORING is REDUCE) then (QUALITYNETMORK is QUA 6. If (EVALUATION is LOMBITERRORRATE) and (MONITORING is REDUCE) then (QUALITYNETMORK is NOC 7. If (EVALUATION is HIGHBITERRORRATE) and (MONITORING is REDUCE) then (QUALITYNETMORK is QUA 8. If (EVALUATION is LOMBITERRORRATE) and (MONITORING is DONOTREDUCE) then (QUALITYNETMORP 9. If (EVALUATION is HIGHBITERRORRATE) and (MONITORING is DONOTREDUCE) then (QUALITYNETMORY 10. If (EVALUATION is HIGHBITERRORRATE) and (MONITORING is REDUCE) then (QUALITYNETMORK is QL 4 If

EVALUATION is and Then $\begin{array}{lll}\text { EVALUATION is } & \text { MONITORING is } & \text { QUALITYNETMNOR } \\ \text { LOMBITERROF - } & \text { DONOTREDUC - } & \text { QUALITYNETV - }\end{array}$ HIGHBITERROF

HIGHBITERROF REDUCE

none

REDUCE

4 III

$\square$ not

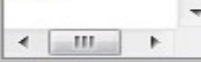

NOQUALITYNE

QUALITYNETV

none

4 III

$\square$ not

- Connection $\neg$ Weight:

or

- and

Add rule

Change rule

Renamed FIS to "GSM"

Figure 5. Rule editor for GSM network

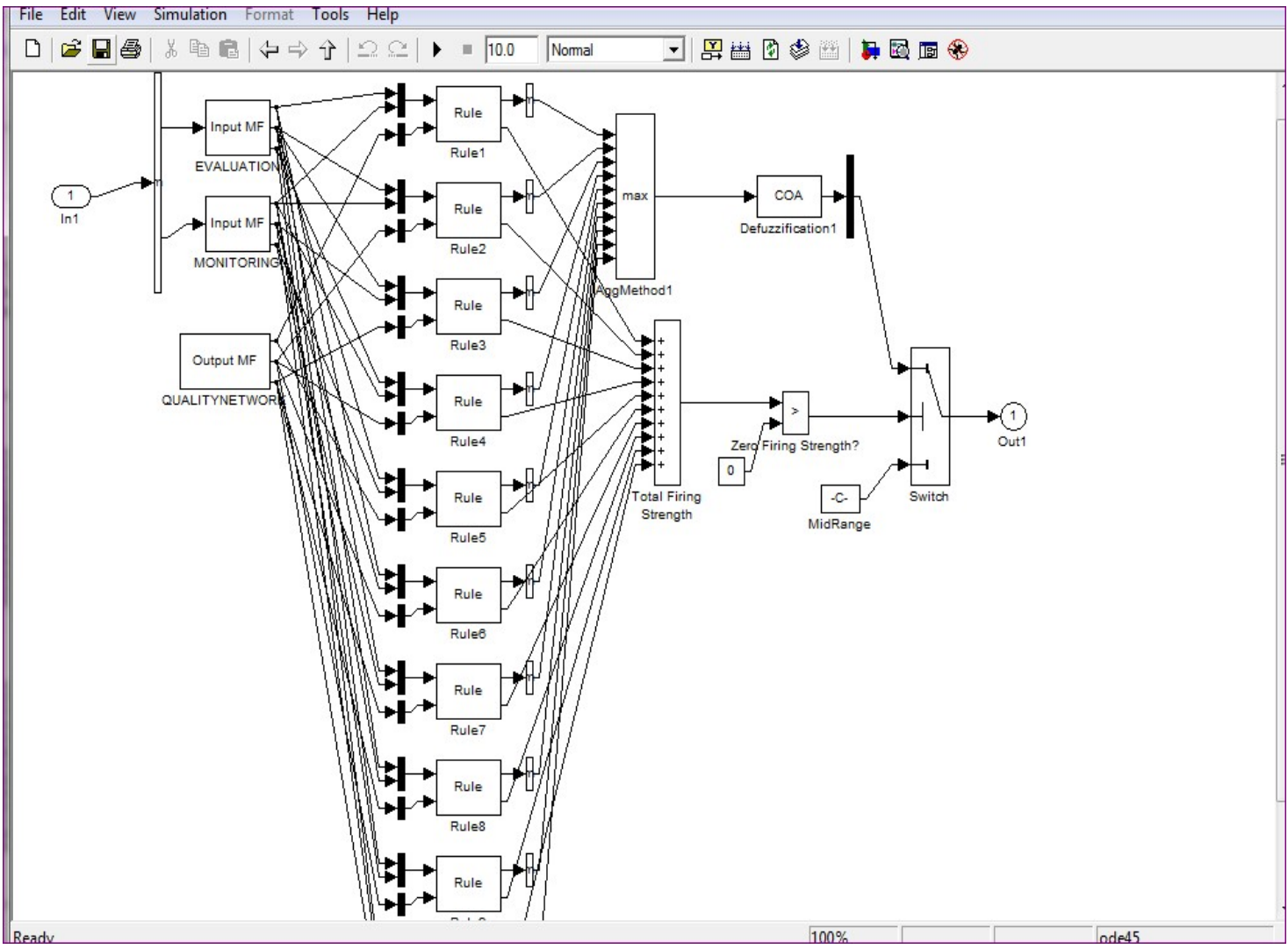

Figure 6. Imbibed rules in the fuzzy logic block 


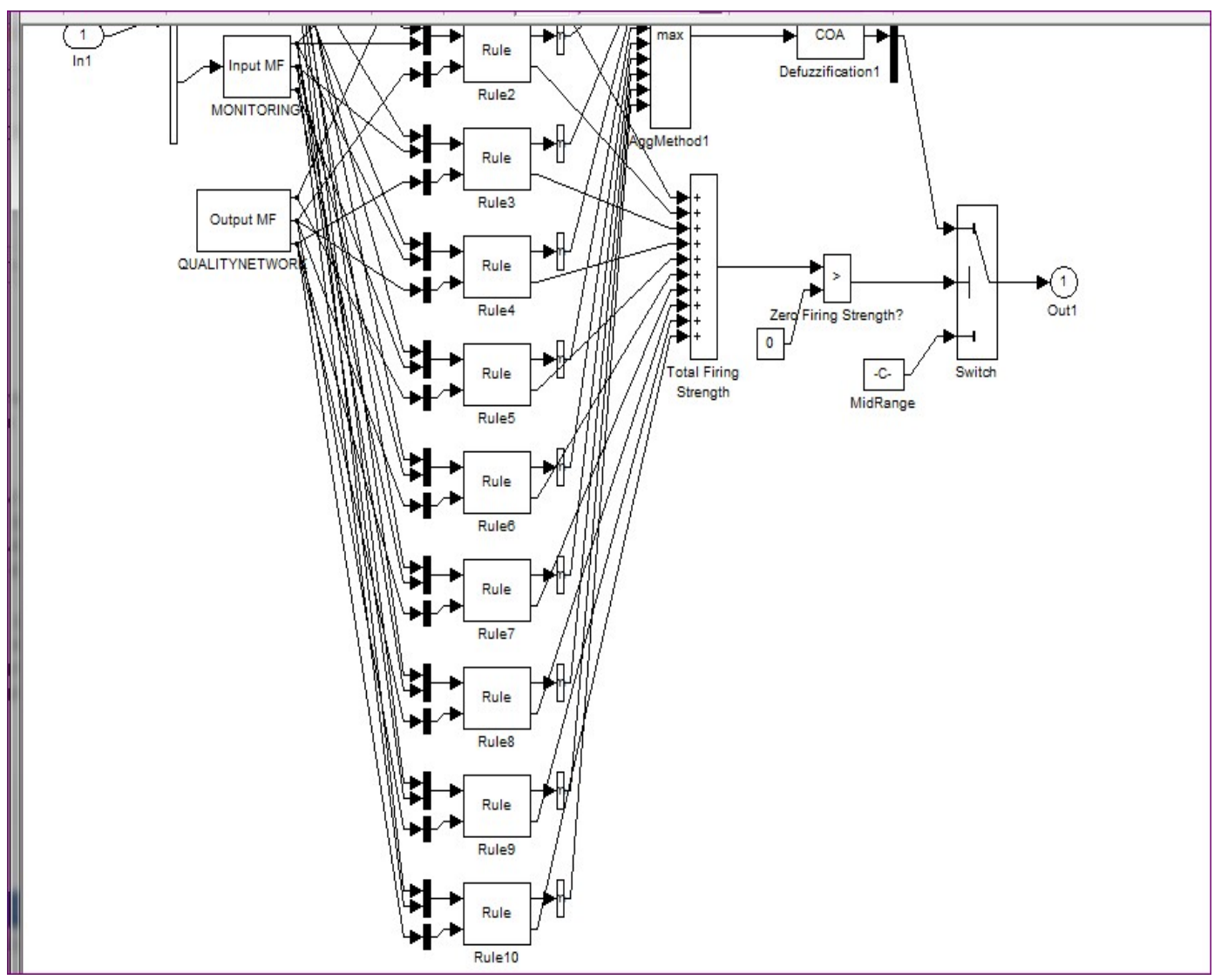

Figure 7. Imbibed GSM rules in the fuzzy logic block 
To design a Simulink model for real time monitoring and evaluation of GSM quality of service using intelligent agent

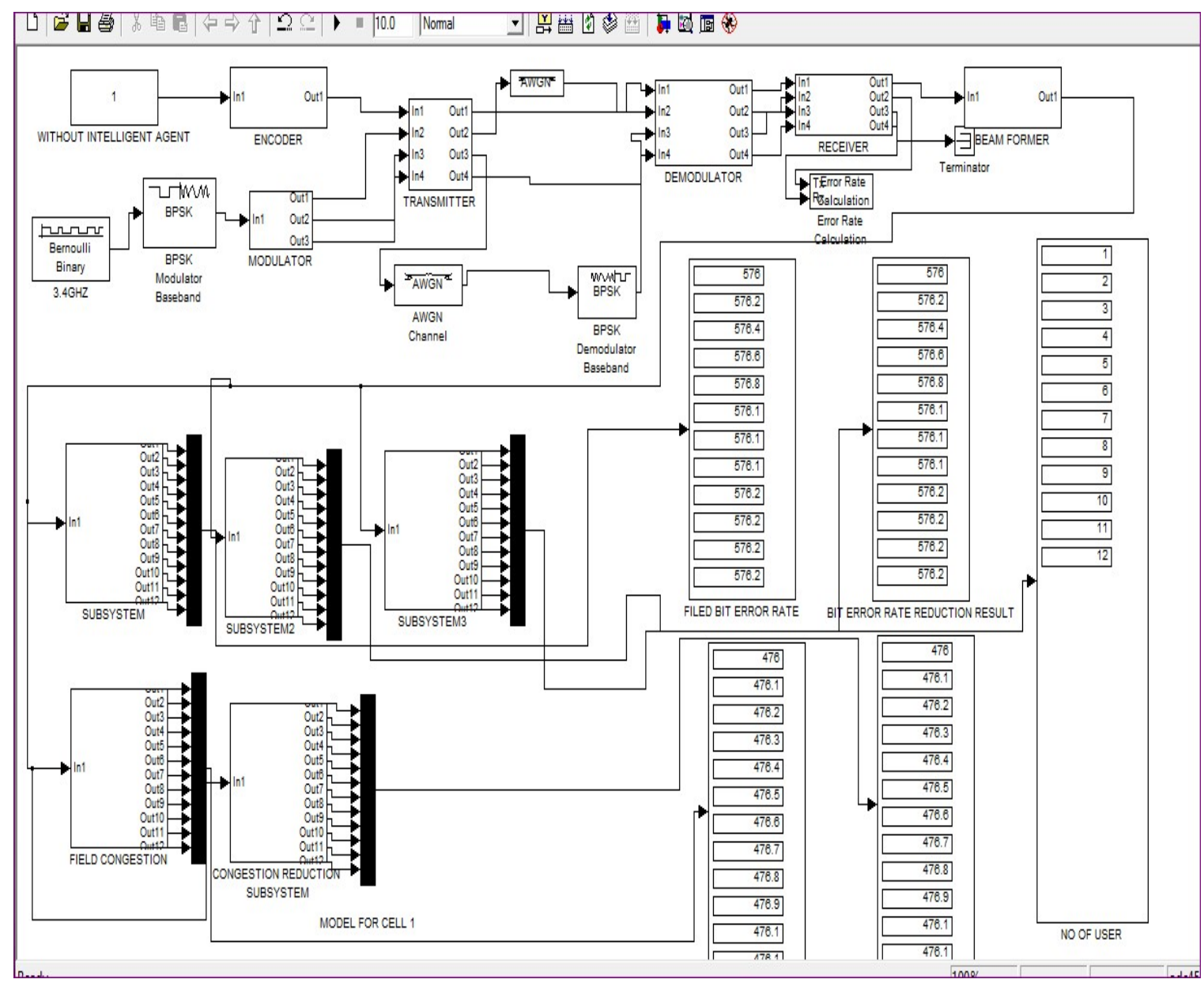

Figure 8. Designed Simulink model for real time monitoring and evaluation of GSM quality of service without using intelligent agent 


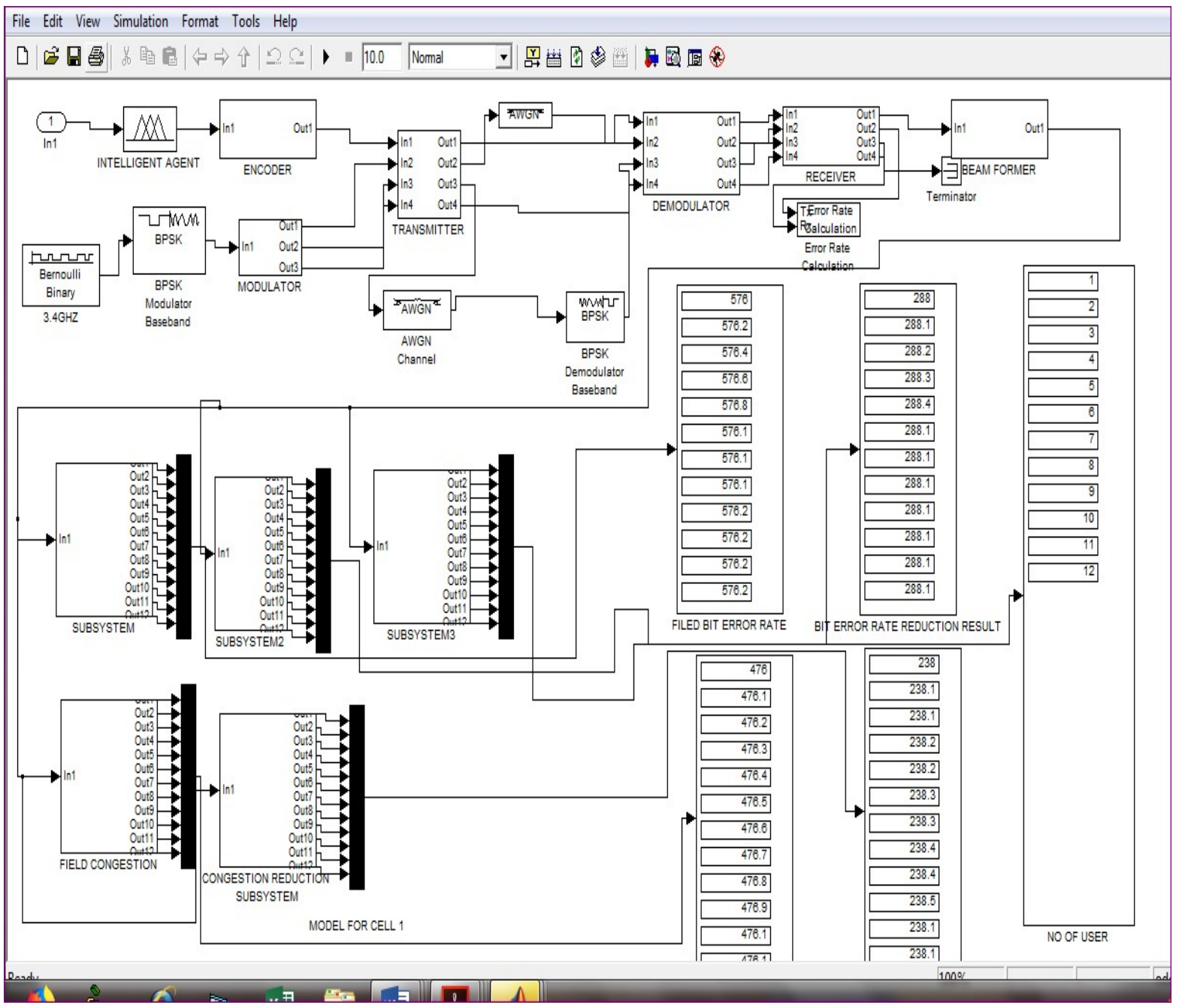

Fig 9 Designed Simulink model for real time monitoring and evaluation of GSM quality of service $\mathrm{t}$ using intelligent agent

\section{Results and Discussion}

The following are the results obtained using the intelligent agent technology in the course of the study. Figure1. Shows Fuzzy inference system editor for GSM that has two inputs of evaluation and monitor. It also has an output of quality network.

Figure 2. shows membership function for GSM evaluation that analyses the bit error rate.

Figure 3. displays the membership function editor for monitoring. This monitors and finds out when the communication network is high and instructs for its reduction.

Figure 4. shows membership function for quality network that identifies if the network is free or not.

Figure 5. shows rule editor for GSM network that is strictly trained to stick to the rule of reducing the bit error rate when it is high for an efficient and quality network.

Figures 6 and 7. show the ten rules of GSM network imbibed in the intelligent agent fuzzy logic control block that enhance the reduction of high bit error rate for an effective and quality network.Figure 8 . is the designed Simulink model for real time monitoring and evaluation of GSM quality of service without using intelligent agent. In this Figure 8. When the bit error rate is high there is an increase in congestion in the communication network thereby leading to low quality or bad network where service will not be experienced Figure 9 Shows 
designed Simulink model for real time monitoring and evaluation of GSM quality of service using intelligent agent.Figure 9. shows that when an intelligent agent monitors and finds out that there is an increase in bit error rate that might cause the network not to be free, the intelligent agent reduces the high bit error rate and the congestion concurrently thereby enhancing quality and free network in communication system. Figure 10 is a comparison of bit error rate without and with intelligent in real time monitoring and evaluation of GSM quality of service. It shows that the high bit error rate reduces when an intelligent agent is incorporated thereby enhances quality service of free communication network to the subscribers. On the other hand there is no free communication network when an intelligent agent is not introduced in the system thereby causing poor network. Figure 11. Shows comparison of congestion without and with intelligent agent in real time monitoring and evaluation of GSM quality of service. Fig 11 shows that there is congestion reduction when an intelligent agent is incorporated in the system which increases the quality of the communication network unlike when an intelligent agent is not incorporated in the system. The channel capacity for GSM quality network was first determined, from the result, the bit error rate for was calculated. An intelligent GSM rule was designed that will monitor and minimize the high bit error rate for quality service and finally designing a Simulink model for real time monitoring and evaluation of GSM quality of service using intelligent agent. The result obtained was $10 \%$ better than using the conventional methods or techniques like Hieratical, Proportional Integral Derivative (PID). Real time monitoring and Evaluation of GSM quality of service using intelligent agent is preferred to other conventional methods as it can guarantee a perfect and free network in our communication network. Table 1. is a Comparison of bit error rate without and with intelligent in real time monitoring and evaluation of GSM quality of service Table 2. Compared the congestion without and with intelligent in real time monitoring and evaluation of GSM quality of service. The result obtained was $10 \%$ better than using the conventional methods or techniques like Hieratical, Proportional Integral Derivative (PID).

Table 1 Comparing bit error rate without and with intelligent in real time monitoring and evaluation of GSM quality of service

\begin{tabular}{|l|l|l|}
\hline $\begin{array}{l}\text { BIT ERROR RATE WITHOUT } \\
\text { INTELLIGENT AGENT }\end{array}$ & $\begin{array}{l}\text { BIT ERROR RATE WITH } \\
\text { INTELLIGENT AGENT }\end{array}$ & TIME \\
\hline 576 & 288 & 1 \\
\hline 576.2 & 288.1 & 2 \\
\hline 576.4 & 288.2 & 3 \\
\hline 576.6 & 288.3 & 4 \\
\hline 576.8 & 288.4 & 5 \\
\hline 576.10 & 288.1 & 6 \\
\hline 576.12 & 288.1 & 7 \\
\hline 576.14 & 288.1 & 8 \\
\hline 576.16 & 288.1 & 9 \\
\hline 576.18 & 288.1 & 10 \\
\hline 576.20 & 288.1 & 11 \\
\hline 576.22 & 288.1 & 12 \\
\hline
\end{tabular}


Table 2 Comparing congestion without and with intelligent in real time monitoring and evaluation of GSM quality of service

\begin{tabular}{|l|l|l|}
\hline $\begin{array}{l}\text { CONGESTION WITHOUT } \\
\text { INTELLIGENT AGENT }\end{array}$ & $\begin{array}{l}\text { CONGESTION WITH } \\
\text { INTELLIGENT AGENT }\end{array}$ & TIME \\
\hline 476 & 238 & 1 \\
\hline 476.1 & 238.1 & 2 \\
\hline 476.2 & 238.1 & 3 \\
\hline 476.3 & 238.2 & 4 \\
\hline 476.4 & 238.2 & 5 \\
\hline 476.5 & 238.3 & 6 \\
\hline 476.6 & 238.3 & 7 \\
\hline 476.7 & 238.4 & 8 \\
\hline 476.8 & 238.4 & 9 \\
\hline 476.9 & 238.1 & 10 \\
\hline 476.10 & 238.1 & 11 \\
\hline 476.12 & 238.1 & 12 \\
\hline
\end{tabular}




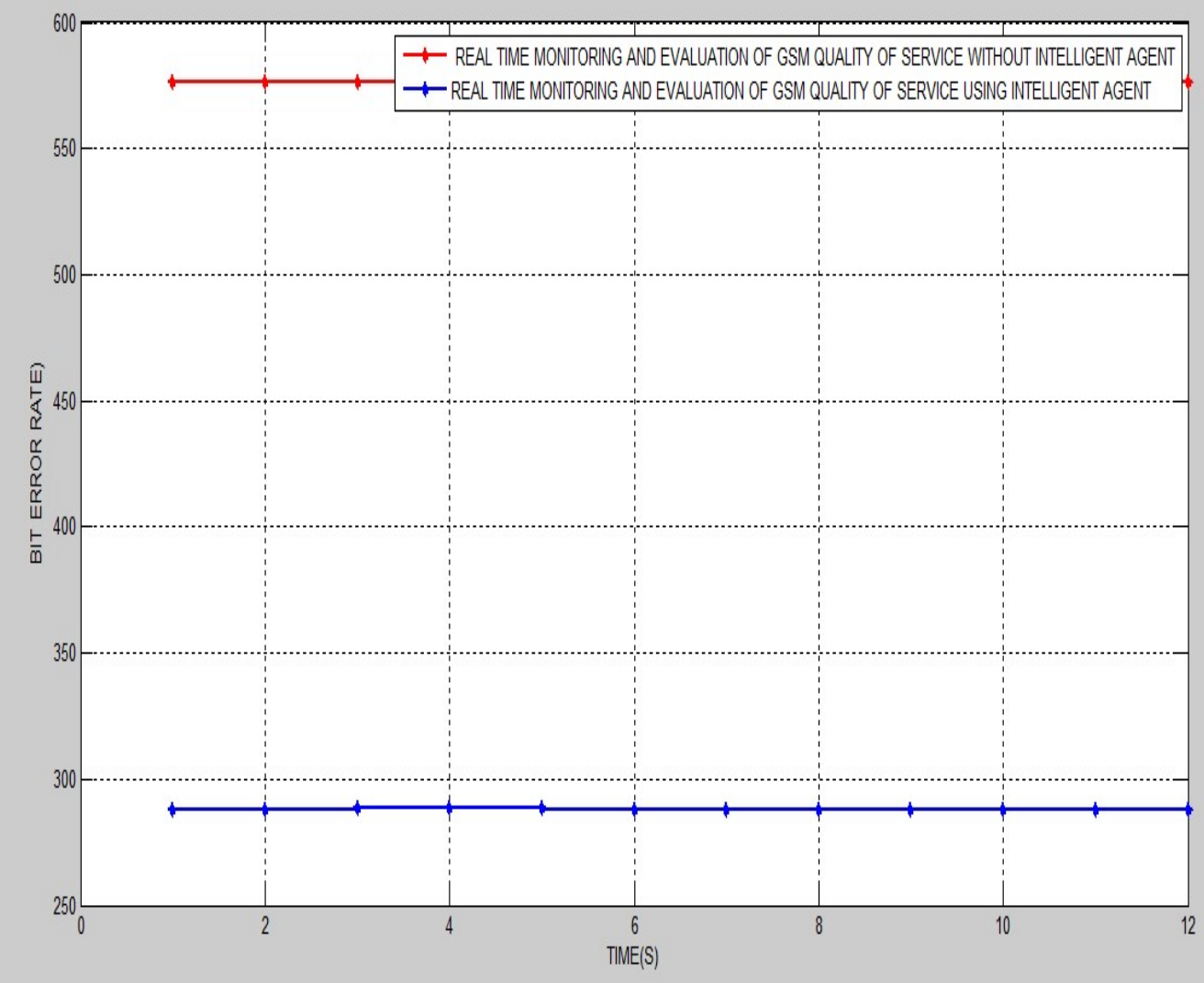

Figure 10 shows a comparison of bit error rate without and with intelligent in real time monitoring and evaluation of GSM quality of service. 


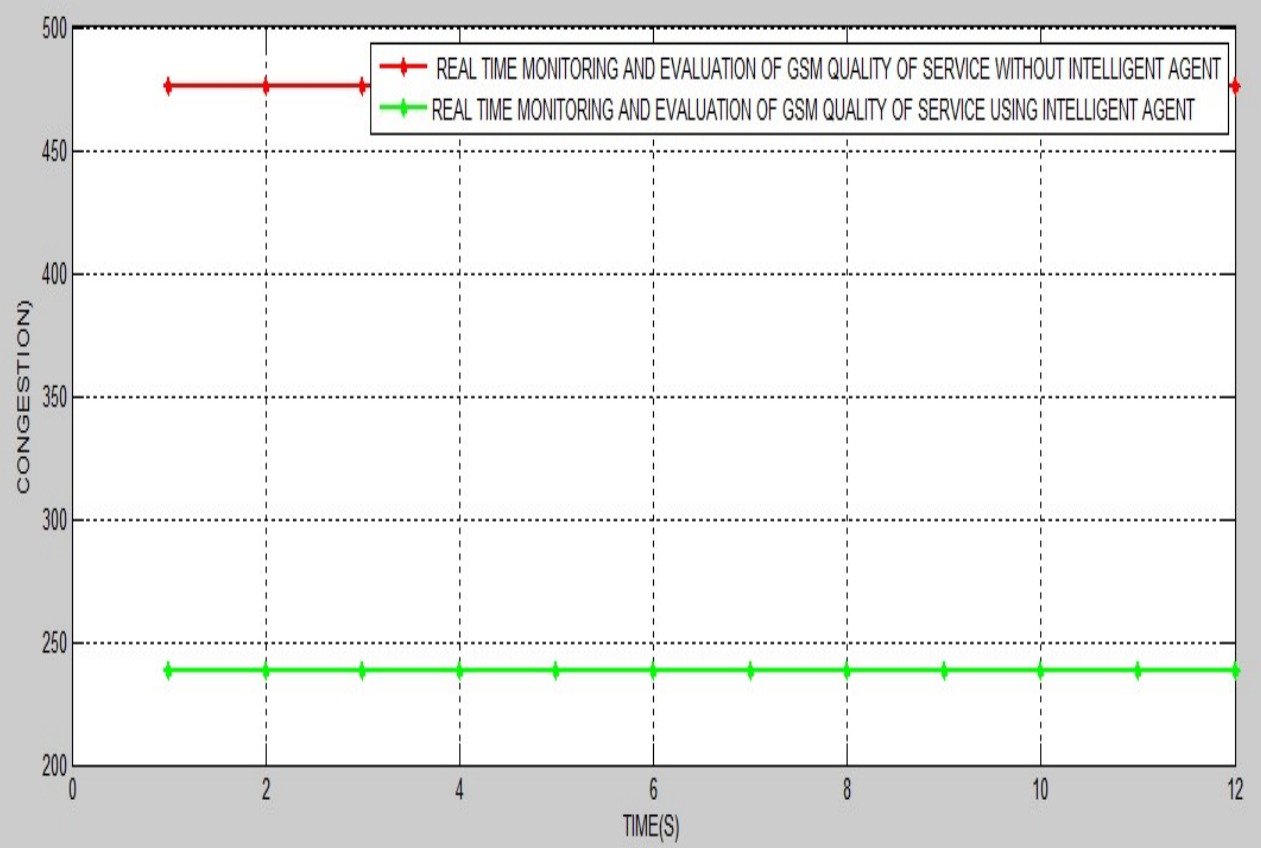

Figure 11. Comparing congestion without and with intelligent agent in real time monitoring and evaluation of GSM quality of service

\section{Conclusion}

The problem of not having free and quality network in our communication network can be overcome by real time monitoring and evaluation of GSM quality of service using intelligent agent. This can be done in this way, determining the channel capacity for GSM quality network, determining the bit error rate for the given capacity, designing an intelligent GSM rule that will monitor and reduce the high bit error rate for quality service and designing a Simulink model for real time monitoring and evaluation of GSM quality of service using intelligent agent The result obtained is a free and quality communication network.

\section{References}

[1] Mani Subramanian,"Network Management: Principles and Practice”, 2008 Edition, Pearson Education

[2] Makki, S., Wunnava, S., 2008. Next Generation Networks and Code Mobility. ISAST Transactions on Communication and Networking, No.1, Vol 2

[3] "Agent based computing: A booklet for executing", http://www.eurescom.de/

[4] Stallings, W., 1999. SNMP, SNMPv2, SNMPv3 and RMON 1 and 2, third ed. Addison Wesley

[5] Yemini, Y., 1993. “The OSI Network Management Model”, IEEE Communications Magazine, vol.31, no.5, pp.20-29. May. 
[6] Stephan, R., Ray, P., Paramesh, N., 2004. Network management platform based on mobile agents. International Journal of Network Management 14, 59-73.

[7] Liotta, A., Pavlou, G. Knight, G., 2002. "Exploiting agent mobility for large-scale network monitoring", IEEE Networks, 16:7-15.

[8] Rubinstein, M.G., Duarte, O., Pujolie, G., 2002. "Scalability of a network management application based on mobile agents", Journal of Communication and Networks, 5:240-248.

[9] Manvi, S.S., Venkataram, P., 2006. Agent based subsystem for multimedia communications. IEEE Proceedings Software Journal 153 (1), 38-48.

[10] Mydhili K Nair, Gopalakrishna V, 2011, Applying Web Services with Mobile Agents For Computer Network Management, International Journal of Computer Networks and Communications (IJCNC), Vol. 3, No. 2, pp 125-144, March.

[11] Weiss (1999) Multi-Agent Systems, MIT Press

[12] Wooldridge, M. (2002) An Introduction tomulti-agent systems. John Wiley \& Sons Limited England, ISBN: 0-471-49691-X

[13] Jennings, N. R., and Wooldridge, M. J. (1998). Application of Intelligent Agent: Foundations, Applications and Markets, pp. 3-28, Secaucus, NJ, Springer-Verlag, Berlin.

[14] Chang, Y. S., Yang, C. T., and Luo, Y. C. (2011) An Ontology based Agent Generation for Information Retrieval on Cloud Environment. J. UCS, 17(8): p. 1135-1160.

[15] Guessoum, Z. andBriot, J. P.(1999).From Active Object to Autonomous Agents", IEEE Concurrency, Volume 7(3), pp. 68-78, July/September.

[16] Boudaoud, K. and Zahia, G. (2000). A Multi-agents System for Network Security Management. Sixth IFIP Conference on Intelligence in Networks (SmartNet'2000), Vienna, Austria, September 18 - 22. 